\title{
Biokompatible Werkstoffe und Bauweisen - Ergebnisse und Ziele
}

\section{Journal Article}

\section{Author(s):}

Wintermantel, Erich

Publication date:

1995

Permanent link:

https://doi.org/10.3929/ethz-b-000422545

Rights / license:

In Copyright - Non-Commercial Use Permitted

Originally published in:

Biomedizinische Technik 40(s1), https://doi.org/10.1515/bmte.1995.40.s1.13 


\section{Biokompatible Werkstoffe und Bauweisen - Ergebnisse und Ziele Erich Wintermantel \\ Lehrstuhl für Biokompatible Werkstoffe und Bauweisen, ETH Zürich, Wagistr. 23, CH-8952 Schlieren}

\begin{abstract}
Einleitung
Biokompatibilität ist als Verträglichkeit eines technischen mit einem biologischen System definiert. Diese Verträglichkeit wird angestrebt unter dem Aspekt der Strukturkompatibilität und dem der Oberflächenkompatibilität. Bestehende Verträglichkeit zwischen diesen beiden Systemen wird bestätigt durch ein Vital-AvitalInterface, das aus enwünschten ortsständigen Zellen und Geweben einerseits und aus einer gewünschten Werkstoffoberfläche und -struktur andererseits besteht. In der Regel wird die Intaktheit eines solchen definierten Interfaces über lange Zeit, häufig für die gesamte Restlebenszeit des Empfängers, angestrebt. Ziel der Forschung und Entwicklung biokompatibler Werkstoffe ist damit die Schaffung definierter und beständiger Interfaces. Dabei ist auch zu denken an Beschichtungen mit degradablen Polymeren auf nicht degradablen Trägerwerkstoffen, um während des Abbaus der Schicht Medikamente, Wachstumsfaktoren, andere bioaktiven Substanzen oder Zellen freizusetzen bis ein optimales VitalAvital-Interface erzeugt ist, das während der angestrebten Zeit im Sinn des Erhalts von Zell- und Gewebetyp stabil bleibt.
\end{abstract}

Das Gejbiet der biokompatiblen Werkstoffe und Bauweisen schliesst neben medizinischen Anwendungen von Werkstoffen und damit dem Humanbiosystem auch Biosysteme der Umwelt ein. In beiden Fällen kann Verträglichkeit des technischen mit dem biologischen System durch gleiche oder ähnliche Werkstoffherstell- und BauteilFertigungs-Technologien erreicht werden und in beiden Fällen soll das biologische System durch die synthetisch geschaffene Struktur nicht geschädigt werden. Damit können unter der Forderung nach Biokompatibilität völlig neue umweltrelevante Massenwerkstoffe entwickelt werden, die dann, in Analogie zu medizinischen Implantaten, als ökotrope Werkstoffe oder Ökoimplantate zu bezeichnen sind.

Der Umgang der involvierten Forscherpersönlichkeiten mit Technologien, die aus einem anderen als dem medizintechnischen Bereich stammen, kann ausserdem zu nützlichen Synergien führen und das klassische medizinische Werkstoffgebiet um neue Impulse bereichern. Sowohl das gezielte Suchen in und das Verstehen von benachbarten Disziplinen als auch die Übertragung in das eigene Forschungsgebiet ist nachhaltig zu fördern.

Weil das Forschungsgebiet der biokompatiblen Werkstoffe und Bauweisen Erfahrungen und Arbeitsweisen sowohl aus technischen Fächern, z. B. dem Maschinenbau, der Elektrotechnik und den Werkstoffwissenschaften, aber auch aus der Medizin, Biologie und Biochemie erfordert, ist es besonders dazu geeignet, interdiziplinäre oder, besser, syndisziplinäre Forschungsstrategien (1) umzusetzen. Es wird beispielsweise als nützlich erachtet, systematisch Nachbardiziplinen, z. B. die Physik, die Chemie, die Informationswissenschaften und die Verfahrenstechnik daraufhin zu überprüfen, ob dort entstandene neue Verfahren und Technologien nicht mit Vorteil auch zur Lösung grundsätzlicher Probleme hier oder zur Verbesserung der Biokompatibilität von Werkstoffen und Bauteilen nutzbar sind. Ein systematisches Innovationsscreening in möglichst vielen Technologiefeldern sollte integraler Bestandteil der Biokompatibilitätsforschung sein.

Das notwendige Zusammenspiel von wissenschaftlichen Disziplinen zeigt sich auch in der Fertigung von Bauteilen aus einem neuen biokompatiblen Werkstoff, in der Neuentwicklung von Prozesstechniken für diesen Werkstoff. Hier wird der Begriff der Bauweisen verwendet, der die Fertigkeiten eines Technologen umschreibt, mit verschiedenen Werkstoffen ein Bauteil möglichst kostengünstig herzustellen. Dies wird angestrebt mit hochintegrierten Bauweisen, um mit wenig Prozessschritten komplexe Bauteile zu erzielen, die zuvor definierte Aufgaben erfüllen.

\section{Strukturkompatibilität}

Darunter ist die optimale, im Falle von medizinischen Implantaten, die möglichst physiologische, Übertragung von Kräften durch das Implantat zu verstehen. Sie ist ein besonderes Merkmal lasttragender 
Implantate, die vor allem in der orthopädischen Chirurgie und in der Unfallchirurgie zur Anwendung gelangen, dazu zählen Prothesen grosser Gelenke, Fixateure und Osteosynthesesysteme.

Nimmt man an, dass alle lasttragenden Strukturen des Körpers anisotrop strukturiert sind und gerichtete Elemente enthalten, $z$. B. kollagene Fasern oder ossäre Trabekel, die in Hauptspannungsrichtung verlaufen, so kann abgeleitet werden, dass dann eine möglichst physiologische Kraftübertragung durch das Implantat stattfindet, wenn auch der Implantatwerkstoff selbst anisotrop strukturiert ist und die auf das Implantat wirkenden Kräfte anlog dem in der Empfängerstruktur herrschenden Situation übertragen werden.

Moderne Implantatentwicklung schliesst neben den Parametern Anisotropie und Dauerfestigkeit auch die folgenden Werkstoffund Bauteileigenschaften ein:

-Freiheit von Metallionen an der Oberfläche, um Beteiligung an einer durch Metallionen vermittelten Allergie zu verhindern,

-erwünschter Grad an Röntgentransparenz, um einerseits das Implantat im konventionellen Röntgenverfahren abbilden zu können, andererseits aber im Strahlengang vor und hinter dem Implantat liegende Empfängerstrukturen beurteilen zu können und

-ein CT- und NMR-kompatibles Verhalten im Sinn von Artefaktfreiheit der abgeleiteten Signale.

Mit diesem für eine Implantatentwicklung um zahlreiche Detailanforderungen reduzierten Pflichtenheft sind sowohl klinische als auch technologische Parameter berücksichtigt.

Fasst man Anisotropie, Fehlen von Metallionen, Röntgentransparenz und CT/NMR-Kompatibilität zusammen, so bieten Kohlenstoffaserverbundwerkstoffe ein hohes Entwicklungspotential $(2,3)$ für strukturkompatible Implantate, vorausgesetzt, die Kohlenstoffasern sind vollkommen von Matrix umschlossen und ein Freiliegen von FaserMatrix-Interfaces ist ebenso ausgeschlossen wie ein Freiwerden von Kohlenstoffasern. Dies muss für kurz-, lang- und endlosfaserverstärkte Polymere gelten. Aus dem Bauteil freigewordene Kohlenstofffasern, die sich im Interstitium befinden, könnten chronische Entzündungsreize verursachen und bei Anschluss an das Lymphsystem in Lymphknoten zu unerwünschten Gewebs- reaktionen führen. Diesem Aspekt muss besondere Beachtung geschenkt werden, weil technische Kohlenstoffasern eine Mindestlänge aufweisen, um einen gerichteten Verstärkungseffekt in der Matrix zu erzielen, die grösser ist als es phagozytierbare Partikel sind.

Andere technisch eingesetzte Fasern, wie Borfasern, Glassfasern oder Aramidfasern scheiden aufgrund ihrer aufwendigen und teuren Bearbeitung (Bor), ihrer mangelnden Korrosionsbeständigkeit (Glass) oder ihrer geringen relativen Druckfestigkeit (Aramid) aus.

Polymere Matrices, die eine vergleichsweise geringe Wasseraufnahme aufweisen, die hohe Beständigkeit gegenüber enzym- und elektrolythaltigen Medien aufweisen und die hohe mechanische Eigenschaften bieten, sind sogenannte Hochtemperaturthermoplaste, z.B. aus der Klasse der Polyetherarylketone (PEAK). Polyetheretherketon (PEEK) ist ein bei etwa $400^{\circ}$ Celsius und honigartiger Konsistenz geschmolzener Thermoplast, der mit Kohlenstoffasern eine sehr gute Haftung eingehen kann und der sowohl in bekannten Herstellverfahren, wie dem Prepregverfahren, oder auch in Entwicklung befindlichen modifizierten Spritzguss- und Fliesspressverfahren verarbeitbar ist. Duroplastische Matrices finden in unseren Entwicklungen an der ETH keine Berücksichtigung für Implantate, da ein prozessabhängiger Gehalt an toxischen Restmonomeren nicht ausgeschlossen werden kann und die Fertigung besondere Sicherheitsmassnahmen erforderlich macht.

Die Entwicklung strukturkompatibler Werkstoffe und Implantate schliesst die Prozessentwicklung für die spätere Fertigung mit ein, denn Werkstoffe erhalten durch den zugehörigen Fertigungsprozess ihre im Bauteil wirksamen Eigenschaften oder vorhandene Eigenschaften werden durch die Fertigung modifiziert. Besonders bei Mehrphasen-systemen, wie den faserverstärkten Polymeren, eröffnet sich eine Vielzahl möglicher Verfahren und Fertigungs-technologien, die, unter Sicherung der nötigen physikalischen und chemischen Eigenschaften des zu prozessierenden Werkstoffs, nach Wirtschaftlichkeitsgesichtspunkten ausgewählt, optimiert oder neuentwickelt werden.

Andere Beispiele für die hohe Prozessabhängigkeit der künftigen Werkstoffeigenschaften sind kurzfaserverstärkte Polymer-Bauteile, darunter eine im Fliesspressverfahren hergestellte kohlenstoff- 
faserverstärkte PEEK-Schraube für die Osteosynthese.

\section{Oberflächenkompatibilität}

Die zum Empfängergewebe hin interfacebildende Schicht des Werkstoffs und Bauteils ist für ein Anwachsen ortsständigen Gewebes von Bedeutung. Diese Schicht kann aus dem Trägerwerkstoff selbst gebildet sein, als Beispiel gelten Titanlegierungen, die durch Spontanpassivierung in oxidischer Umgebung Titandioxid bilden und das eine ausgezeichnete Oberflächenkompatibilität ergibt. Jedoch sind auch Schichten entwickelt worden, die das Empfängergewebe in seinem anorganischen Teil im Sinn eines Werkstoffmimikry nachbilden. Dazu zählt Hydroxylapatit, ein schwer lösliches Pentacalciumphosphat, das beispielsweise in Plasmaspritzverfahren (4) mit oder ohne Vakuum verarbeitbar ist und eine den Trägerwerkstoff maskierende keramische Schicht bildet. Die Verarbeitung im genannten Verfahren erfordert eine sehr präzise Prozesssteuerung, um einerseits chemische Umwandlungen des Schichtwerkstoffs während des Auftrags auf den Trägerwerkstoff $z u$ verhindern und andererseits den Trägerwerkstoff durch das Plasma nicht thermisch zu zersetzen.

Abscheiden aus übersättigter Lösung (5) ist ein Weg, um bei erheblich. niedrigeren Temperaturen, als Plasmaverfahren sie erfordern, Hydroxylapatit auf polymere Träger aufzubringen, und damit einen kohlenstoffaserverstärkten Hüftendoprothesenischaft im proximalen Teil zu beschichten mit dem Ziel der späteren Osseointegration.

Kombinierte Oberflächen- und Strukturverträglichkeit, Tissue Engineering (6)

Ein Sonderfall der Implantatentwicklung stellt ein angiopolarer Zellträger $(7,8)$ dar, bei dem die Strukturkompatibilität nicht der Übertragung von Kräften dient sondern dem Bilden eines differenzierten Blutgefässmusters. Ob Kräfte eine Rolle spielen bei der Ausbildung eines Blutgefässmusters in Implantatnähe, beispielsweise induziert durch kleinste sich kontrahierende Narbenzüge, die im Rahmen einer bindegewigen Einkapselung entstehen können, ist noch zu klären. Es konnte in Implantationsversuchen an der Ratte gezeigt werden, dass Blutgefässe in der Grössenordnung von Kapillarien an konvexen
Stellen eines Implantats gebildet werden können und grössere Blutgefässe sich an Konkavitäten bilden, im Sinn eines "Gefässnabels".

Ein solcher konkav-konvex gestalteter Zellträger wurde für die spätere Transplantation von Hepatozyten, zunächst aus resorbierbaren Polymeren, später aus nicht resorbierbarer Aluminiumoxidkeramik hergestellt. Es bestand zunächst die Vorstellung, ein metabolisch aktives Ersatzorgan, ein Organoid, wie es transplantierte Hepatozyten bilden sollen, benötige lediglich während der wenige Wochen dauernenden Einwachsphase ein polymeres Gerüst im Sinne einer Leitschiene, das später jedoch die vollständige Ausbildung eines Ersatzorganes störe und daher am besten aus resorbierbarem Werkstoff zu fertigen sei. Jedoch führten die in vitro beobachteten ph-Wertveränderungen während des Abbaus des Polymers und die in vivo gesehene Zurücklassung von Kristalliten dazu, einen nicht abbaubaren Werkstoff zu wählen. Für die Bauteilfertigung wurde ein eigenes Verfahren entwickelt, bei dem ein keramischer Schlickertropfen auf eine vorgewärmte, mit Kavitäten versehene Metallplatte getropft wird und durch Dampfbildung sich eine keramische Blase bildet, die platzt und eine offene Hohlkugel hinterlässt und die nach dem Brand eine definierte Wandporosität aufweist. Die Grösse der Hohlkugeln wurde so gewählt, dass sie mit grossen Kanülen ( 9 gauge) injizierbar sind. Derzeit wird an einer Miniaturisierung der Zellträgerstruktur gearbeitet, um die Implantation mit möglichst atraumatischen oder minimal invasiven Techniken zu ermöglichen.

Ein anderer Sonderfall, in dem Oberflächenund Strukturkompatibilität unter dem Aspekt der Werkstoffentwicklung nicht getrennt betrachtet werden können, stellt ein Bandscheibenersatzwerkstoff dar, der zum interbody fusion element weiterentwickelt wird (9). Dort führt eine Phasenseparation während des Aushärtens eines Knochenzementes dazu, dass sich ein offenporiger und inkompressibler Schaum bildet, in den ortsständiges Gewebe einwachsen kann.

In Entstehung begriffene Technologien, darunter vor allem Methoden des tissue engineering nutzen Oberflächen- und Strukturverträglichkeit biokompatibler Werkstoffe, um aus kultivierten Zellen Gewebe wachsen zu lassen. Unter tissue engineering ist die gezielte Einflussnahme auf das Wachstumsverhalten von Geweben 
durch Werkstoffe zu verstehen. Dieses Gebiet lässt besonders für den funktionellen Organersatz und die Entwicklung vitaler Testsysteme innovative Entwicklungen erwarten.

\section{Wirtschaftlichkeit}

Soll eine Werkstoff- oder Implantatentwicklung insofern erfolgreich sein, dass sie später zahlreichen Patienten zur Verfügung steht oder marktbildend wirkt, so muss sie sich der Wirtschaftlichkeitsprüfung unterziehen lassen. Häufig scheiterten in der Vergangenheit Neuentwicklungen biokompatibler Werkstoffe daran, dass die für eine spätere Kommerzialisierung aufzuwendenden Geldmittel unabsehbar hoch zu werden drohten und Entwickler sowie Sponsoren ihre Aktvitäten einstellten, als lediglich Substanzen im Labormasstab zur Verfügung standen. Schliesslich müssen Kosten für eine Zulassung nach FDA- oder Regeln anderer Behörden berücksichtigt werden. Unter diesen Gesichtspunkten muss der Forscher, der biokompatible Werkstoffe entwickelt, vor allem bei der Entwicklung zugehöriger Prozesstechniken der Wirtschaftlichkeit besondere Aufmerksamkeit zuwenden. Hohe Entwicklungskosten einerseits und das Risko des Werkstoff- und Bauteilversagens andererseits stehen einer Produktentwicklung mit hoher Wertschöpfung gegenüber.

Eine pragmatische Beurteilung gaben Galletti et al. (10) am Beispiel von Organersatzwerkstoffen: "The denial of the unknown, which has reached paranoid proportions in some celebrated cases of device failure, will have to be replaced by a sober appreciation of what can and cannot be achieved in organ replacement, regardless of progress in technology". Diese Betrachtung und die daraus abzuleitenden Handlungsmaximen empfehlen sich für alle Neuentwicklungen biokompatibler Werkstoffe.

\section{Literatur}

(1) Wintermantel, E.: Teile und das Ganze: Syndisziplinarität. Bulletin. Magazin der ETH Zürich (251) 32 - 34 (1993).

(2) Mayer, J., Ruffieux, K., Ha, S.-W., Tognini, R., Koch, B., Wintermantel, E.: Knitted carbon fiber reinforced biocompatible structures: A new biomaterial for load bearing implants with homoelasticity to bone. Soc. Biom. Trans. 1994, 230.
(3) Widmer, M., Isler, J., Callenbach, T., Fröhlich, M., Meier, D., Mayer, J., Wintermantel, E., Tschanz, P., Lüthi, H., Mazenauer, K., Klostermann, L.: Entwicklung eines anisotropen Hüftendoprothesenschaftes. Verfahren und Technologien. Oberflächen und Werkstoffe 5, 34 - 37 (1995).

(4) Ha, S.-W., Mayer, J., Koch, B., Wintermantel, E.: Plasma-sprayed hydroxylapatite coating on carbon fiber reinforced thermoplastic composite materials. J. Mat. Sc. Materials in Medicine , 481 - 484 (1994).

(5) Ha, S.-W., Reber, R., Eckert, K.-L., Mayer, J., Wintermantel, E.: Precipitation of hydroxylapatite coatings in simulated body fluids: A novel technology for coating carbon fiber reinforced thermoplastic composites. Proceedings of the XXIth Annual Congress of the European Society for Artificial Organs, Barcelona, 1994 / Journal of Artificial Organs 17,430 (1994).

(6) Wintermantel, E., Mayer, J., Blum, J., Eckert, K.-L., Lüscher, P., Mathey, M.: Tissue engineering scaffolds using superstructures. Biomaterials. Special issue about tissue engineering, in press.

(7) Wintermantel, E., Cima, L., Schloo, B., Langer, R.: Angiopolarity of cell carriers: directional angiogenesis in resorbable liver cell transplantation devices. In: Angiogenesis: key principles, science technology, medicine. R. Steiner et al. (eds), Birkhäuser Verlag Basel, 1992, 331 - 334.

(8) Eckert, K.-L., Rösler, U., Mathey, M., Mayer, J., Winetrmantel, E.: Open porous ceramics for cell transplantation devices: a new biomaterial - first results. In: Bioceramics vol. 6, Ducheyne P. and Christiansen, D., (eds), Butterworth-Heinemann, 1993 , $251-255$.

(9) Mathey, M., Meier, C., Hottenstein, F., Mayer, J., Wintermantel, E.: A new intervertebral disc respacing biomaterial: open porous bone cement. Proceedings of the Monte Verita conference 1993 on biocompatible materials systems, 1993 , $89-91$.

(10) Galletti, P. M., Aebischer, P., Lysaght, M. J.: The dawn of biotechnology in artificial organs. ASAIO Journal (1995); 49-57. 